\title{
Associação dos antígenos leucocitários humanos com a ausência de resposta humoral à vacina da hepatite B em pacientes renais crônicos hemodialisados
}

\author{
Association of humans leucocitary antigens with humoral nonresponsive to \\ hepatitis B vaccine in chronic hemodialysis patients
}

\author{
Joaquim Xavier de Sousa Júnior ${ }^{1}$, José Tibúrcio do Monte Neto ${ }^{2}$, José Adail Fonseca de Castro ${ }^{3}$, \\ Hélida Monteiro de Andrade ${ }^{3}$ e Semiramis Jamil Hadad do Monte ${ }^{3}$
}

\begin{abstract}
RESUMO
A vacinação com antígeno de superfície do vírus da hepatite B não tem eficácia satisfatória em pacientes hemodialisados. O objetivo do estudo foi investigar uma possível associação entre antígenos leucocitários humanos e a baixa capacidade de produção de anticorpos protetores (anti-HbS) contra o antígeno de superfície do vírus da hepatite B em pacientes renais crônicos de programa de hemodiálise. Os antígenos HLA DR e DQ foram determinados em 76 pacientes hemodialisados por meio da técnica clássica de microlinfocitotoxicidade. Os resultados demonstraram que 34,2\% dos pacientes eram não-respondedores à vacina VHB. As especificidades HLA mais freqüentes foram: HLA-DR3, DR7 e DQ2, com associação significante para a especificidade HLA-DR3 (p=0,0025; OR 5, 1; IC95\% 1,36-19, 10). Estes dados sugerem a associação dos genes HLA de classe II com a incapacidade de resposta humoral à vacina VHB.
\end{abstract}

Palavras-chaves: Hepatite B. Hemodiálise. Antígeno leucocitário bumano.

\section{ABSTRACT}

Vaccination using surface antigen from hepatitis B virus has not been successfully responded by hemodialysis patients. The present study was aimed at assessing a possible relationship between buman leukocyte antigens and the low production of protective antibodies (anti-HbS) against the surface antigen from hepatitis B by patients with chronic renal failure submitted to bemodialysis programs. The antigens HLA-DR and HLA-DQ were identified in 76 hemodialysis patients through classic microlymphotoxicity. Our results showed that $34.2 \%$ of the patients were non-responsive to the vaccine VHB. The most frequent HLA specificity were: HLA-DR3, $D R-7$ and DQ2 with a significant association for HLA-DR3 ( $p=0.0025$; OR 5.1; IC 95\% 1.36-19.10). Such data suggest an association between genes from HLA class II antigens and the humoral non-response to the vaccine VHB.

Key-words: Hepatitis B. Non-response. Human leucocyte antigen.

Os pacientes portadores de insuficiência renal crônica (IRC), submetidos à hemodiálise (HD), são ainda considerados como população de alto risco para a infecção aguda pelo vírus da hepatite B (VHB), apesar da adoção generalizada das medidas universais recomendadas para a prevenção da transmissão de infecções virais ${ }^{3}$, da vacinação rotineira contra o VHB nos pacientes suscetíveis e da redução das necessidades de hemotransfusão nas unidades de hemodiálise após o emprego terapêutico de eritropoetina. Estudos demonstram que a incapacidade de produzir títulos de anticorpos protetores (NR), em resposta ao estímulo antigênico da vacina do VHB é encontrada em 5 a 10\% dos indivíduos na população geral e, esta resposta inadequada é consideravelmente maior, na faixa de 20 a 40\%, entre os pacientes submetidos à $\mathrm{HD}^{2} 471022$. Esta incapacidade resulta tanto de uma disfunção dos componentes da imunidade natural e adquirida, comprovada in-vitro $^{8}$, como pelas evidências clínicas de imunodeficiência, observada pela elevada suscetibilidade à infecção, responsável por altos índices (36\%) de mortalidade na IRC ${ }^{1325}$.

1. Universidade Federal do Piauí, Teresina, PI. 2. Clínica Nefrológica do Piauí, Teresina, PI. 3. Laboratório de Imunogenética e Biologia Molecular da Universidade Federal do Piauí, Teresina, PI.

Endereço para correspondência: Drª Semiramis Jamil Hadad do Monte. Laboratório de Imunogenética e Biologia Molecular/UFPI. Bloco 16, 64049-550 Teresina, PI

Fax: $5586215-5690$

e-mail:libpi@ufpi.br

Recebido para publicação em 20/6/2003

Aceito em 10/11/2003 
Esforços têm sido feitos para elucidar este fenômeno de imunodeficiência aos estímulos vacinais, com particular ênfase aos estudos com os genes da região do complexo principal de histocompatibilidade, os quais iniciam e controlam a resposta imune efetiva aos antígenos protéicos ${ }^{12}$. Os estudos de associação têm demonstrado uma freqüência elevada dos alelos DRB1*03 e DRB1*07 entre as pessoas NR à vacina $\mathrm{VHB}^{1}{ }^{19}$, embora ainda seja controversa a contribuição destes genes como reguladores da baixa capacidade de resposta aos estímulos vacinais.

0 objetivo do presente estudo foi determinar se há associação entre os genes antígeno leucocitário humano (HLA) de classe II com a ausência de resposta humoral à vacina $\mathrm{VHB}$, em pacientes renais crônicos do programa de HD de uma unidade típica de Teresina, Piauí.

\section{MATERIAL E MÉTODOS}

Foram estudados 76 pacientes portadores de insuficiência renal crônica terminal em procedimento hemodialítico convencional, numa unidade típica de HD em Teresina. Os critérios de inclusão foram: estar em programa de HD há mais de 6 meses, quadro clínico estável, esquema completo de vacina VHB (OMS) e mensuração da concentração de anticorpo protetor à vacina VHB (anti-HBs) após conclusão do esquema vacinal. Foram considerados como NR os pacientes com concentração de anticorpo anti-HBs $<10 \mathrm{mIU} / \mathrm{ml}$. 0 estudo foi previamente aprovado pelo Comitê de Ética em Pesquisa da Universidade Federal do Piauí, e os casos analisados forneceram consentimento informado para o pesquisador.

Tipificação antígeno leucocitário humano. A tipificação HLA foi determinada pela técnica padrão de microlinfocitotoxicidade mediada por complemento, utilizando anticorpos monoclonais comerciais (One Lambda Inc, Canoga Park, USA) após obtenção de linfócitos B por separação com partículas imunomagnéticas.

Análise estatística. As freqüências dos antígenos HLA DR e DQ observados nos pacientes NR foi comparada àquelas dos pacientes respondedores (R). A significância da associação foi testada, utilizando o teste do qui-quadrado e o grau de associação foi assegurado pelo cálculo do Odds Ratio, com IC de 95\%.

\section{RESULTADOS}

Dos 76 pacientes estudados, $65,8 \%$ foram classificados como respondedores à vacina $\mathrm{VHB}$ e 34,2\% como não-respondedores. A Tabela 1 apresenta as freqüências antigênicas das especificidades HLA DR e DQ dos pacientes NR e R à vacina VHB. Entre os pacientes NR, observou-se freqüência elevada das especificidades: HLA-DR3 (31\%x8\%), DR7 (34,6\%x30\%) e DQ2 (46\%x32\%). 0s OR observados para HLA DR3, DR7 e DQ2 foram respectivamente de 5,$1 ; 1,23$ e 1,42, sendo a diferença entre os grupos estatisticamente significante apenas para a especificidade HLADR3 ( $p=0,0025, I C=1,36-19,10)$. Dos pacientes NR, portadores do fenótipo HLA-DQ2, 46\% expressavam o haplótipo [HLADR3,DQ2] e 46\% o haplótipo [HLA-DR7, DQ2].
Tabela 1- Freqüência das especificidades HLA-DR e DQ para os pacientes não-respondedores e respondedores à vacina vírus da hepatite $B$, em programa de bemodiálise crônica, de uma unidade bemodialítica de Teresina, Piauí.

\begin{tabular}{lrr}
\hline & \multicolumn{2}{c}{ Pacientes $(\%)$} \\
\cline { 2 - 3 } Especificidades HLA & $\mathrm{NR}(\mathrm{n}=26)$ & $\mathrm{R}(\mathrm{n}=50)$ \\
\hline DR1 & 31,0 & 14,0 \\
DR3 & 7,7 & 26,0 \\
DR4 & 34,6 & 30,0 \\
DR7 & 3,8 & 14,0 \\
DR8 & 11,5 & 4,0 \\
DR9 & 11,5 & 2,0 \\
DR10 & 15,0 & 14,0 \\
DR11 & 7,7 & $\mathrm{ND}$ \\
DR12 & 26,9 & 42,0 \\
DR13 & 15,0 & 8,0 \\
DR14 & 23,0 & 20,0 \\
DR15 & $\mathrm{ND}$ & 10,0 \\
DR16 & 46,0 & 32,0 \\
DQ2 & 15,0 & 14,0 \\
DQ4 & 23,0 & 14,0 \\
DQ5 & 42,0 & 48,0 \\
DQ6 & 23,0 & 21,0 \\
DQ7 & 11,5 & 26,0 \\
DQ8 & 11,5 & 8,0 \\
DQ9 & &
\end{tabular}

ND: não determinado

\section{DISCUSSÃO}

No presente estudo observou-se um aumento significante da freqüência da especificidade HLA DR3 nos pacientes NR à vacina VHB. Esta especificidade foi identificada em 31\% dos NR e em $8 \%$ dos $R(p=0,0025)$. 0 cálculo do OR associado com DR3 foi de 5,1, significando que os indivíduos com esse fenótipo são 5,1 vezes mais suscetíveis à não ativar a produção de anticorpo anti-HbS ao estímulo antigênico da vacina VHB, que os pacientes portadores de outras especificidades HLA de classe II. Neste mesmo grupo de pacientes as freqüências antigênicas de HLA DR7 e DQ2 foram elevadas, mas a associação foi fraca e, portanto não atingiu significância estatística. Em contraste com os dados da literatura, não foi observada nenhuma associação para a especificidade HLA DR4, e inclusive para esta população estudada de NR, a freqüência

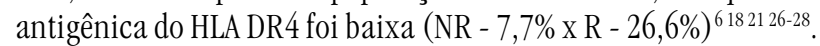

Considerando que as especificidades HLA DR3 e DR7 estão freqüentemente em desequilíbrio de ligação (DL) com DQ2 e para os pacientes estudados observou-se que $92 \%$ dos NR apresentavam estes haplótipos, torna-se pertinente questionar se estes genes seriam de fato marcadores de suscetibilidade dos pacientes à reduzida capacidade de produção de anticorpos anti-HbS após o estímulo vacinal. Outros estudos da literatura, com os mesmos objetivos e em diferentes grupos étnicos, têm demonstrado associação com estas especificidades, sugerindo que estes genes codificadores das moléculas HLA-DR3, HLA-DR7 e HLA-DQ2 seriam genes de suscetibilidade à resposta inadequada à vacina VHB e não apenas um gene em DL com o antígeno HLA-DQ2 ${ }^{2324}$.

Da mesma forma, McDermott e cols ${ }^{1417}$ têm demonstrado que os genótipos HLA DRB1*07 e DQB1*02 prevalentes nos pacientes 
NR à vacina VHB, também estão associados com a incapacidade de resposta humoral à nova formulação vacinal, contendo como determinantes antigênicos as frações pré-S1, pré-S2 além do clássico antígeno de superfície do VHB.

Diante disto, embora o complexo principal de histocompatibilidade represente a região genômica mais freqüentemente associada com a ausência de resposta humoral à vacina do VHB, o mecanismo exato desta associação é desconhecido ${ }^{512}$. Nos pacientes portadores de IRC em HD esta situação é mais complexa. Além dos fatores imunogenéticos associados a esta incapacidade de resposta, os fatores metabólicos secundários ao estado urêmico não podem ser afastados $^{911}$.

A tipificação HLA, pré-vacinação, dos pacientes renais crônicos com determinação dos haplótipos HLA DR3-DQ2 ou HLA DR7-DQ2, pode ser uma estratégia epidemiológica útil na identificação de pacientes com maior probabilidade de não responder à vacina do VHB. Nestes pacientes, como medida profilática para diminuir o risco de infecção aguda pelo VHB, sugere-se implementação de programa de controle das infecções virais nas unidades de $\mathrm{HD}^{3}$ e no futuro, a utilização de novas formulações vacinais e ou imunomoduladores como terapêutica adjuvante da vacina. Estes procedimentos contribuiriam para a redução da prevalência de pacientes hemodialisados portadores crônicos do vírus da hepatite B, os quais no Brasil ainda correspondem a 5\% da população em programa de hemodiálise ${ }^{20}$.

\section{AGRADECIMENTOS}

À Clínica Nefrológica do Piauí (CLINEFR0) e o seu corpo de funcionários por permitir o desenvolvimento deste trabalho com os pacientes em programa de hemodiálise.

\section{REFERÊNCIAS BIBLIOGRÁFICAS}

1. Alper CA, Kruskall MS, Marcus-Bagley D, Craven DE, Katz AJ, Brink SJ, Diestang JL, Awdeh Z, Yunis EJ. Genetic prediction of nonresponse to hepatitis B vaccine. The New England Journal of Medicine 321: 708-712, 1989.

2. Chang PC, Schrander-van der Meer AM, van Dorp WT, van Leer E. Intracutaneous versus intramuscular hepatitis $B$ vaccination in primary non-responding haemodialysis patients. Nephrology Dialysis Transplantation 11:191,1996.

3. Consultant Meeting to Update Recommendations for the Prevention and Control of Bloodborne and Other Infections Among Chronic Hemodialysis Patients. Recommendations for Preventing Transmission of Infections Among Chronic Hemodialysis Patients. Morbidity and Mortallity Weekly Report 27:1-43, 2001.

4. Craven DE, Awdeh ZL, Kunches LM, Yunis EJ, Diesstag JL, Werner BG, Polk F, Snydman DR, Platt R, Crumpacker CS, Grady GF, Alper CA. Nonresponsiveness to hepatitis $B$ vaccine inhealth care workers. Annals of Internal Medicine 105:356360,1986 .

5. Desombere Y, Gijbels A, Verwulgen Leroux-Roels G. Characcteriztion of the T cell recognition of hepatitis B surface antigen (HbsAg) good and poor responders to hepatitis vaccines. Clinical Experimental of Immunology 122:390-399,2000.

6. Desombere I, Willems A, Leroux-Roels G. Response to hepatitis B vaccine: multiple HLA genes are involved. Tissue Antigens 51: 593-604,1998.

7. Dienstag JL, Werner BG, Polk F, Snydman DR, Craven DE, Platt R, Crumpacker CS. Hepatitis B vaccine in health care personnel: safety, immunogeneicity, and indicators of efficacy. Annals of Internal Medicine 101:34-40,1984.
8. Girnat M, Sester M, Sester U, Kaul H, Köhler H. Molecular aspects of T and B cell function in uremia. Kidney International 59: S206-\$211, 2001.

9. Jacbson IM, Jaffers G, Dienstag JL, Tolkoff-Rubin NE, Cosimi AB, Delmonico F, Watkins E, Hinkle C, O'Rourke S, Russel PS, Rubin RH. Immunogenicity of hepatitis B vaccine in renal transplant recipients. Transplantation 39:393-395, 1985.

10. Jungers P, Devillier P, Salomonm H, Cerisier JE, Courouce AM. Randomised placebo-controlled trial of recombinant interleukine-2 in chronic uraemic patients who are non-responders to hepatitis B vaccine. Lancet 344:856, 1994.

11. Kaul H, Girndt M, Sester U, Sester M, Köhler H: Initiation of hemodialysis treatment leads to improvement of $\mathrm{t}$ cell activation in patients with end stage renal disease. American Journal of Kidney Disease 35:611-616, 2000.

12. Klein J, Sato A. The HLA System. The New England Journal of Medicine 343:782786,2000 .

13. Mailloux LU, Bellucci AG, Wilkes BM, Napolitano B, Mossey RT, Lesser M, Bluestone PA. Mortality in dilysis patients: Analyses of the causes of death. American Journal of Kidney Disease 3:326-335,1991.

14. McDermott AB, Cohen SB, Zuckerman JN, Madrigal JA. Hepatitis B thirdgeneration vaccines: improved response and conventional vaccine non-responseevidence for genetic basis in humans. Journal Viral Hepatology 5 (supl 2): 9-11, 1998.

15. McDermott AB, Cohen SB, Zuckerman JN, Madrigal JA. Human leukocyte antigens influence the immune response to a pre-S/S hepatitis B vaccine. Vaccine 28 ; 330-339, 1999

16. McDermott AB, Madrigal JA, Sabin CA, Zuckerman JN, Cohen SB, The influence of host factors and immunogenetics on limphocyte response to Hepagene vaccination. Vaccine 17: 1329-1337, 1999.

17. McDermott AB, Zuckerman JN, Sabin CA, Marsh SG, Madrigal JA. Contribution of human leukocyte antigens to the antibody response to hepatitis B vaccination. Tissue antigens 50: 8-14, 1997.

18. Mineta M, Tanimura M, Tana T, Yssel H, Kashiwagi S, Sasazuki T. Contribution of HLA class I and class II alleles to the regulation of antibody production to hepatitis B surface antigen in humans. International Immunology 8:525531,1996 .

19. Pol S, Legendre C, Mattlinger B, Berthelot P, Kreis H. Genetic basis of nonresponse to hepatitis B vaccine in hemodialyzed patients. Journal Hepatology 11:385-387, 1990.

20. Sesso R. Inquérito epidemiológico em unidades de diálise do Brasil. Jornal Brasileiro de Nefrologia 22 (3-supl 2): 23-26, 2000.

21. Stachowski J, Kramer J, Füst G, Maciejewski C, Baldamus A, Petrayi GG. Relationship between the reactivity to men $B$ virus vaccination and the frequency of MHC class I and II alleles in haemodialysis patients. Scandinavian Journal of Immunology 42:60-65,1995.

22. Stevens CE, Alter HJ, Taylor PE, Zang EA, Harley EJ, Szmuness W Hepatitis B vaccine in patients receiving hemodialysis: immunogenicity and efficacy. The New England Journal of Medicine 311: 496-501, 1984.

23. Svejgasard A, Platz P, Ryder LP. HLA and disease 1982 - a survey. Immunological Review 70:193-218, 1983.

24. Svejgasard A, Platz P, Ryder LP. HLA and disease associations: detecting the strongest association. Tissue Antigens 43:18-27, 1994.

25. US Renal Data System. USRDS 1998 annual data report. American Journal of Kidney Disease 32:S5-S12,1998.

26. Varla-Leftherioti M, Papanicolaou M, Spyropoulu M, Vallindra H, Tsiroyianni P, Tassopoulos N, Kapasouri H, Stavropoulos-Giokas C. HLAassociated non-responsiveness to hepatitis $B$ vaccine. Tissue Antigens 35:60-63,1990.

27. Watanabe H, Matsuchita K, Nobuhiro K, Hirayami K, Okumura M, Sasazuki T. Immunesuppresion gene on HLA-Bw4-DR4-DRw53 haplotype controls nonresponsiveness in humans to hepatitis B surface antigen via CD8+ supressor T cells. Human Immunology 22:9-14,1988.

28. Watanabe H, Okumura M, Hirayama K, Sasazuki T. Hla-Bw54-Dr4-DRw53DQw4 Haplotype Controls Nonresponsiveness To Hepatitis-B Surface Antigen Via CD8-Positive Supressr T Cells. Tissue Antigens 36:69-74,1990. 